\title{
Estenosis mitral no reumática. Complejo de Shone en un paciente adulto
}

\section{Víctor Darú*}

Luciano Fallabrino**

María Noelia Pérez ${ }^{* * *}$

Marcelo Cohen*

* Cardiodiagnóstico Investigaciones Médicas. Buenos Aires. Argentina

** Sección Ecocardiografía. Sanatorio de la Trinidad Mitre. Buenos Aires. Argentina

***Ecografía Vascular. Hospital Italiano. Buenos Aires. Argentina

Recibido:25/05/2020

Aceptado: 20/02/2021

En línea: 30/04/2021

Citar como: Daru V. Fallabrino L, Perez M, Cohen M, Estenosis mitral no reumática. Complejo de Shone en un paciente adulto . Rev Ecocar Pract (RETIC). 2021 (Abril); 4 (1): 22-25. doi: 10.37615/retic.v4n1a9.

Cite this as: Daru V, Fallabrino L, Perez M, Cohen M., Non-rheumatic mitral stenosis. Shone complex in an adult patient. Rev Ecocar Pract (RETIC). 2021 (Abril); 4 (1): 22-25. doi: 10.37615/retic.v4n1a9.

\section{Palabras clave}

$\triangleright$ Complejo de Shone

$\triangleright$ Válvula mitral en paracaídas

\section{Keywords}

$\triangleright$ Shone complex

$\triangleright$ Parachute mitral valve

\section{RESUMEN}

Se presenta el caso de una mujer de 34 años, sintomática por disnea de esfuerzos de un año de evolución, en donde se realiza el diagnóstico de complejo de Shone. Esta afección se caracteriza por presentar una serie de anomalías congénitas obstructivas, o potencialmente obstructivas, en el corazón izquierdo. Inicialmente fueron descritas cuatro afecciones: el anillo supravalvular mitral, la válvula mitral en "paracaídas", la estenosis subaórtica y la coartación aórtica. Sin embargo, en la práctica clínica la definición del complejo ha sido ampliada para incluir otras formas adicionales de obstrucción en el corazón izquierdo.

\begin{abstract}
We present the case of a 34-year-old woman, with symptomatic dyspnea since last year, in which the patient is diagnosed with Shone's Complex. This condition is characterized by a series of obstructive, or potentially obstructive, congenital abnormalities in the left heart. Initially, four lesions were described: mitral supravalvular ring, parachute mitral valve, subaortic stenosis and coarctation of the aorta. Nowadays, the definition of the complex has been expanded to include other additional left obstructive lesions.
\end{abstract}

\section{Presentación del caso}

Mujer 34 años, sin factores de riesgo cardiovascular, con disnea en clase funcional NYHA I - II, de un año de evolución.

Derivada para realizarse un ecocardiograma transesofágico en evaluación por enfermedad valvular.

Al examen físico no presentaba latidos patológicos, los ruidos cardíacos eran normales y se detectó un suave soplo diastólico en el foco mitral, sin chasquido de apertura.

En el ecocardiograma transtorácico los diámetros y espesores de las cuatro cavidades eran normales, la fracción de eyección del ventrículo izquierdo se encontraba preservada sin presentar asinergias regionales.

La válvula mitral presentaba apertura parcialmente restringida con acortamiento subvalvular (Videos 1 y 2).
Las cuerdas tendinosas se encontraban moderadamente engrosadas y desde ambas valvas confluyen en un solo músculo papilar. Estos hallazgos son sugestivos de estenosis mitral congénita por válvula en paracaídas. El flujo mitral presentaba un área de aliasing diastólico a nivel del anillo y otros más turbulentos a nivel de las cuerdas tendinosas (Video 3).

El gradiente medio mitral era de $5,9 \mathrm{~mm} \mathrm{Hg}$ a 90 latidos por minuto (Figura 1).

Desde la vista supraesternal se evidenció una coartación aórtica luego de la emergencia de la subclavia izquierda (Videos 4 y 5 ) con gradiente a predominio sistólico y de baja velocidad $(2,2 \mathrm{~m} / \mathrm{s})$.

Desde vistas transesofágicas se observaba un espolón levemente protruyente en la cara auricular de ambas valvas mitrales, sugestivo de anillo supramitral (Videos 6-8; Figuras 2 y 3 ).

La asociación de anomalías (válvula mitral en paracaídas, anillo supramitral y coartación aórtica) sugerían que se trataba de un complejo de Shone. 


\section{Estudio por imagen}

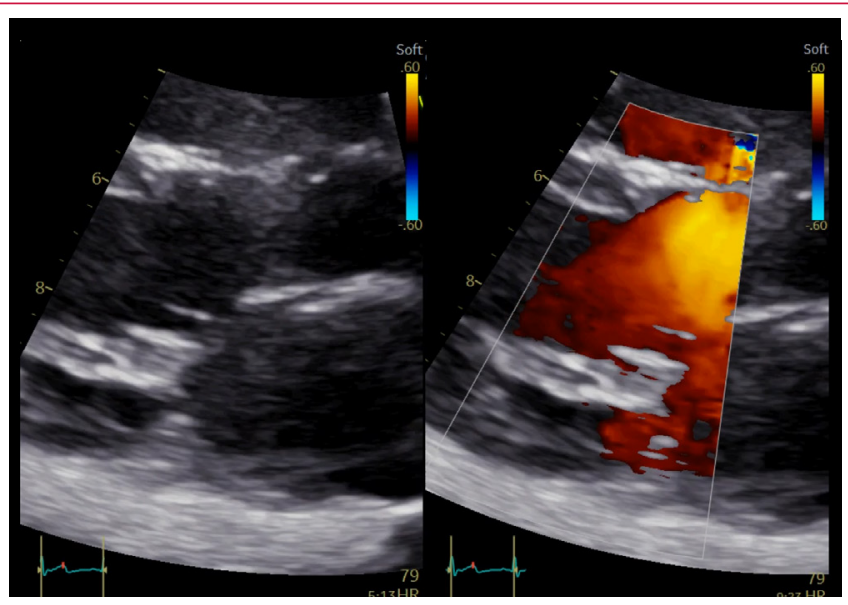

Vídeo 1. Apertura mitral parcialmente restringida, con acortamiento subvalvular y un flujo mitral con un área de aliasing diastólico a nivel del anillo y otros más turbulentos a nivel intercordal

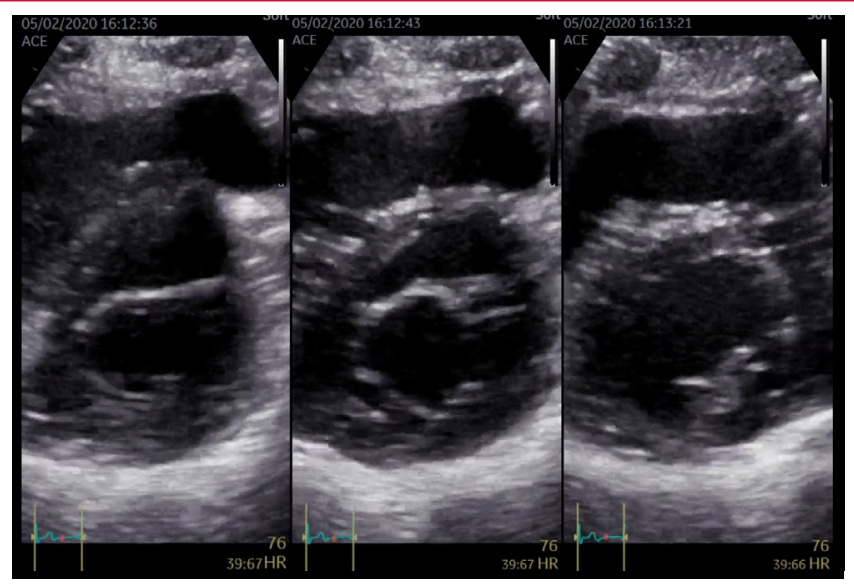

Vídeo 2. Ejes cortos sucesivos desde el anillo. Se observa que el cono mitral confluye en un músculo papilar en posición lateral

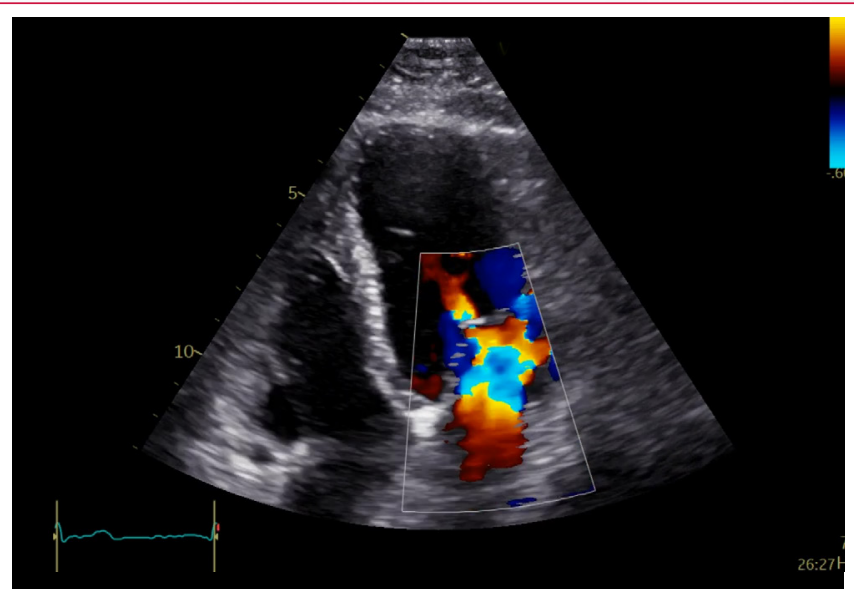

Vídeo 3. El Doppler color demuestra turbulencia a nivel del cono mitral con un llenado ventricular a través de orificios entre las cuerdas tendinosas, sin la imagen habitual "en llama" de la estenosis mitral reumática, ni el flujo como "bigote" del doble orificio mitral

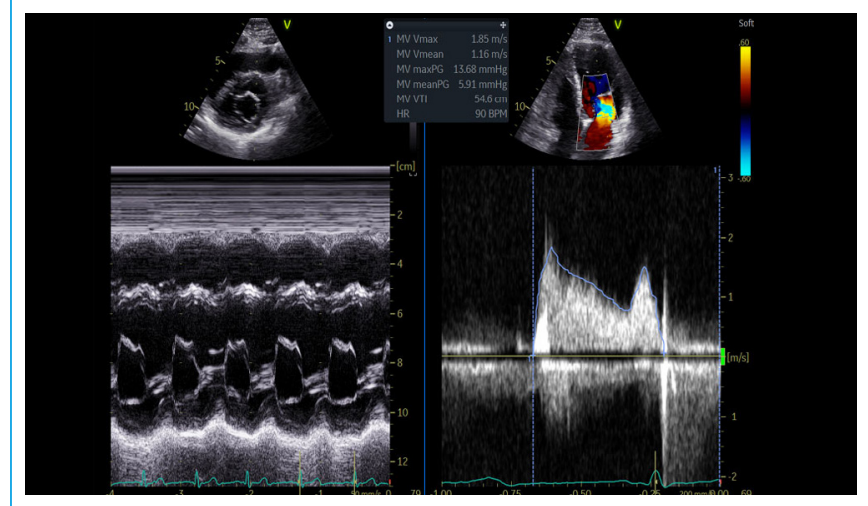

Figura 1. La valva posterior es oponente, sin fusión comisural. El gradiente medio en reposo es de $5,9 \mathrm{mmHg}$

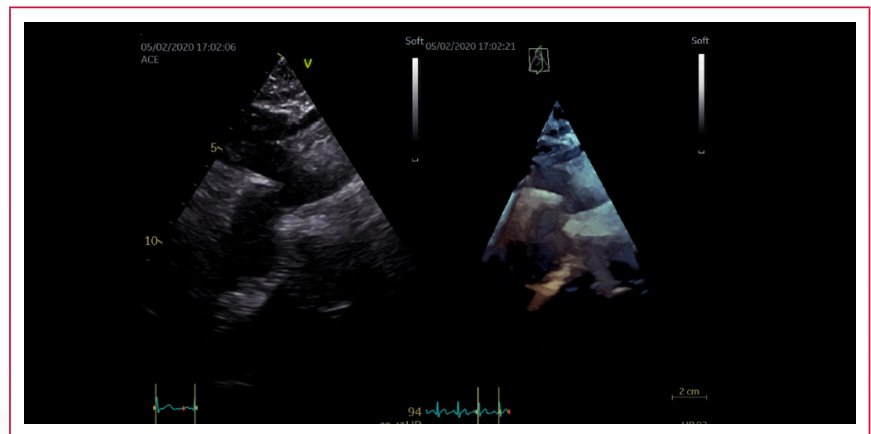

Vídeo 4. Vista supraesternal del istmo aórtico con espolón protruyente luego de la subclavia izquierda

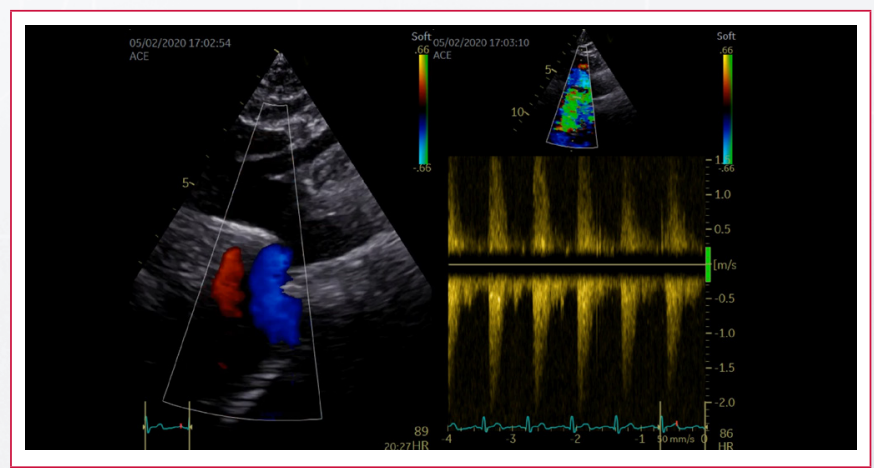

Vídeo 5. Flujo turbulento con gradiente moderado y rampa diastólica incipiente

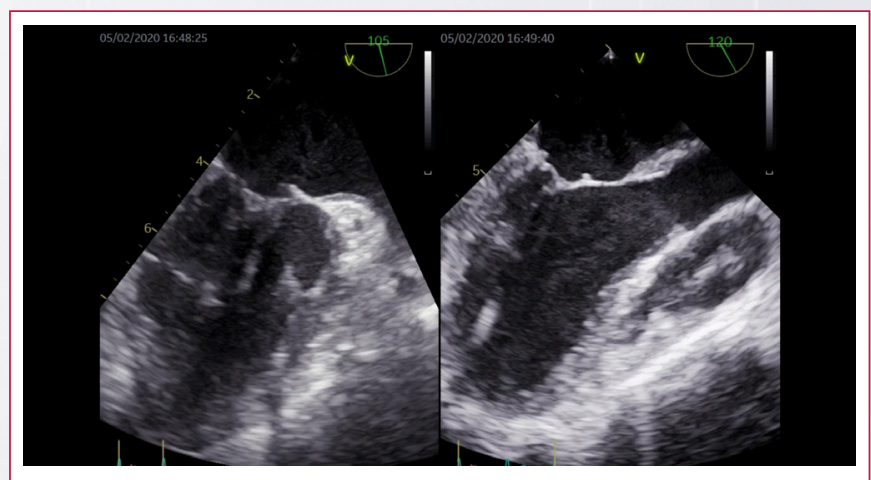

Vídeo 6. Vistas transesofágicas. Se observa la apertura de las valvas no restringidas y la anomalía del aparato subvalvular. En el sector medio de la valva anterior se observa un espolón levemente protruyente, sugestivo de anillo supramitral (en este caso es intramitral). 


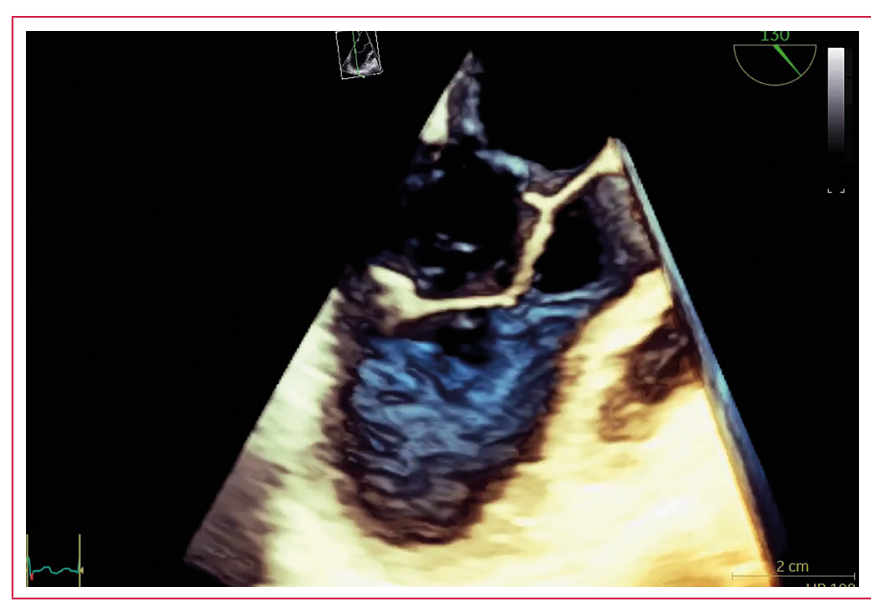

Vídeo 7. Vista tridimensional (3D) del aparato subvalvular mitral y del anillo supramitral.

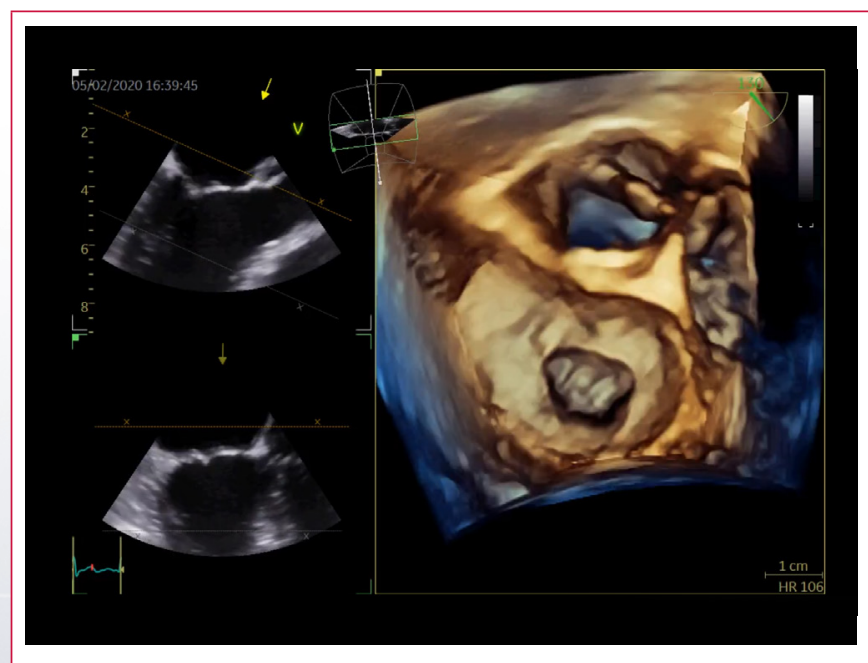

Vídeo 8. Vista 3D de la válvula mitral desde la aurícula izquierda, demostrando el anillo mitral. La válvula aórtica es trivalva, sin evidencia en otras vistas de obstrucción al tracto de salida

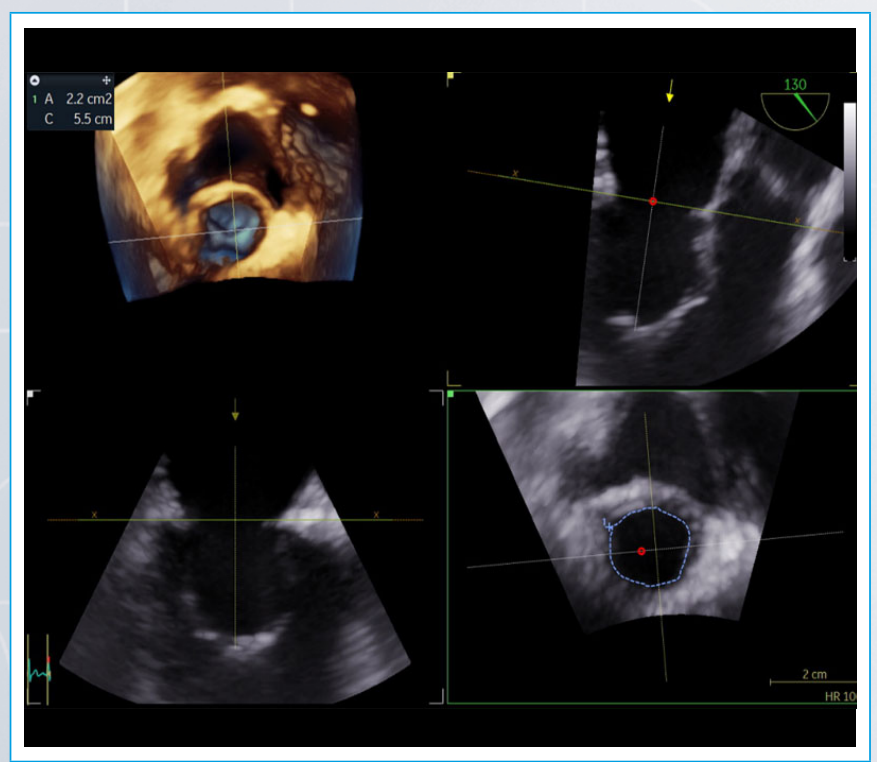

Figura 2. Planimetría del cono mitral por encima del anillo supramitral (área de $2,2 \mathrm{~cm}^{2}$ ).

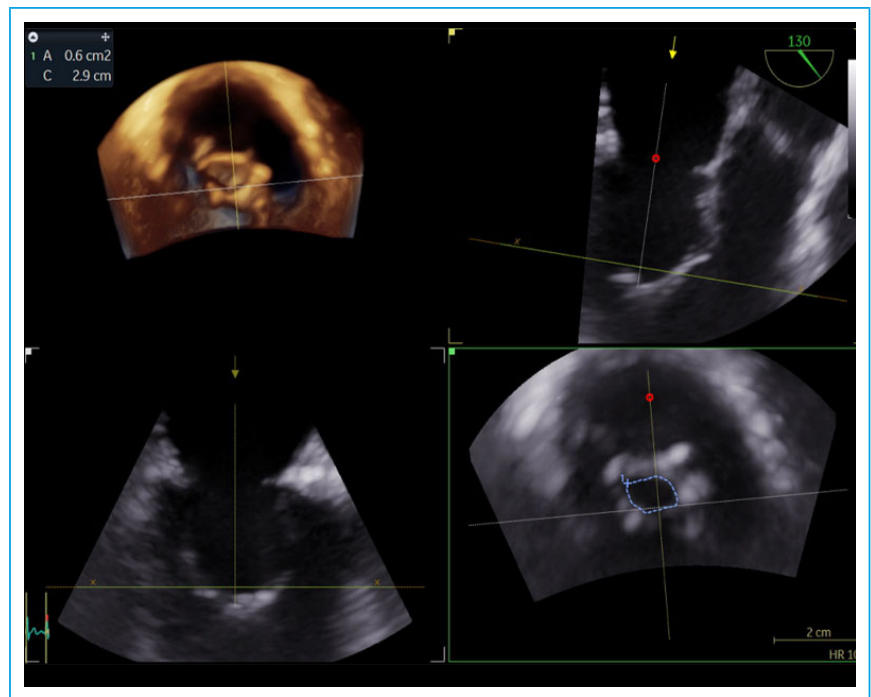

Figura 3. Planimetría a nivel del extremo del cono mitral, estenótico (área $\left.0,6 \mathrm{~cm}^{2}\right)$

\section{Discusión}

John Shone y colaboradores publicaron en 1963 una serie de casos en donde coexistían una variedad de anomalías congénitas obstructivas, o potencialmente obstructivas del corazón izquierdo. Éstas fueron: anillo supravalvular mitral, válvula mitral en "paracaídas", estenosis subaórtica, y coartación aórtica. De los 8 casos reportados, sólo 2 exhibían simultáneamente todas estas condiciones (complejo de Shone completo), mientras que los restantes presentaron al menos dos de ellas (complejo de Shone incompleto) $)^{(1,2)}$.

La válvula mitral "en paracaídas" presenta un solo músculo papilar en donde se insertan las cuerdas tendinosas de ambas valvas. Las cuerdas usualmente son cortas y gruesas, y su inserción papilar convergente restringe la movilidad de los velos. El llenado ventricular se produce entre espacios intercordales que generalmente limitan el libre flujo de sangre conformando una estenosis subvalvular $r^{(1,3,4,5)}$.

El anillo supravalvular mitral es un rodete de tejido conectivo que se origina en la base de la superficie auricular de las valvas, inmediatamente por encima del plano del anillo y protruye dentro de la cavidad. Presentan gradientes significativos el $44 \%$ y rara vez se lo describe en forma aislada ${ }^{(1,3)}$

\section{La estenosis subaórtica puede ser membranosa o tuneliforme ${ }^{(1)}$}

La coartación aórtica suele ser yuxtaductal, a veces sin gradiente significativo $^{(1)}$.

La definición del complejo ha sido ampliada para incluir formas adicionales de obstrucción, como otras lesiones mitrales (estenosis mitral congénita típica, estenosis mitral hipoplásica, cuerdas tendinosas subdesarrolladas con obliteración de los espacios intercordales), aórticas (aorta bicúspide) y estenosis supraaórticas (membrana supra-aórtica o hipoplasia del arco) ${ }^{(2,3)}$.

Es requisito diagnóstico la detección de al menos dos lesiones obstructivas, una mitral y la otra en la salida del flujo del ventrículo izquierdo, ya sea a nivel subaórtico, aórtico o supra-aórtico ${ }^{(6)}$. La prevalencia de cada una de éstas lesiones según las series se detallan en la Tabla $\mathbf{1}^{(4)}$. 


\begin{tabular}{|c|c|c|c|c|c|c|c|c|}
\hline & \multirow[b]{2}{*}{$\begin{array}{l}\text { Pacientes } \\
\text { (n) }\end{array}$} & \multicolumn{3}{|c|}{$\begin{array}{l}\text { Obstrucción del tracto de entrada } \\
\text { del ventrículo izquierdo }\end{array}$} & \multicolumn{3}{|c|}{$\begin{array}{l}\text { Obstrucción del tracto de salida } \\
\text { del ventrículo izquierdo }\end{array}$} & \multirow[b]{2}{*}{$\begin{array}{l}\text { Mortalidad } \\
\text { quirúrgica }\end{array}$} \\
\hline & & $\begin{array}{l}\text { Anillo supra } \\
\text { mitral }\end{array}$ & $\begin{array}{l}\text { Parachute } \\
\text { mitral }\end{array}$ & $\begin{array}{l}\text { Estenosis } \\
\text { mitral } \\
\text { congénita } \\
\text { típica }\end{array}$ & $\begin{array}{l}\text { Estenosis } \\
\text { subaórtica }\end{array}$ & $\begin{array}{l}\text { Válvula aórtica } \\
\text { bicúspide }\end{array}$ & $\begin{array}{l}\text { Coartación } \\
\text { aórtica }\end{array}$ & \\
\hline $\begin{array}{l}\text { Shone } \\
\text { (1963) }\end{array}$ & 8 & $100 \%$ & $50 \%$ & - & $100 \%$ & - & $50 \%$ & - \\
\hline $\begin{array}{l}\text { Bolling } \\
(1990)\end{array}$ & 30 & $73 \%$ & $87 \%$ & - & $93 \%$ & - & $29 \%$ & $14 \%$ \\
\hline $\begin{array}{l}\text { Brauner } \\
(1997)\end{array}$ & 19 & $47 \%$ & $63 \%$ & $25 \%$ & $79 \%$ & - & $68 \%$ & $27 \%$ \\
\hline $\begin{array}{l}\text { Brown } \\
(2005)\end{array}$ & 27 & $41 \%$ & $37 \%$ & $52 \%$ & $59 \%$ & $89 \%$ & $67 \%$ & $11 \%$ \\
\hline $\begin{array}{l}\text { Aslam } \\
(2016)\end{array}$ & 28 & $7 \%$ & $36 \%$ & $93 \%$ & $39 \%$ & $71 \%$ & $75 \%$ & - \\
\hline
\end{tabular}

Tabla 1. modificada de Brown JW, Ruzmetov M, Vijay P, et al. Operative results and outcomes in children with Shone's anomaly. Ann Thorac Surg. 2005 Apr;79(4):1358-65.

A su vez, éste complejo se relaciona con otras anomalías cardíacas, como el ductus arterioso persistente (33-42\%), comunicación interventricular (44\%) e interauricular (33\%), vena cava superior izquierda persistente (21\%) y la obstrucción en el tracto de salida del ventrículo derecho ${ }^{(3,4,6)}$.

Esta entidad representa sólo el $0.6 \%$ de todas las cardiopatías congénitas y la edad media del diagnóstico es 32 años $^{(6)}$. Las manifestaciones clínicas suelen progresar con el paso del tiempo y cerca del 50\% de los pacientes en edad adulta han presentado hospitalizaciones de causa cardiovascular, principalmente debido a insuficiencia cardíaca o arritmias. Si bien la mortalidad es baja en pacientes que han alcanzado la edad adulta, en 92\% requiere algún tipo de intervención y el cerca de la mitad necesita al menos dos a lo largo de su vida.

El procedimiento más prevalente en una primera instancia es el tratamiento de la coartación aórtica (61\%), mientras que el tratamiento quirúrgico o percutáneo de la válvula mitral el de mayor frecuencia en una segunda etapa $(40 \%)^{(6)}$.

La diversidad y la naturaleza compleja de las anomalías cardíacas hacen que sea necesario individualizar el tratamiento y el plan quirúrgico. Los principales marcadores pronósticos postoperatorios son la severidad de la valvulopatía mitral y la presencia de hipertensión pulmonar ${ }^{(3)}$.

\section{Conclusión}

El complejo de Shone es una cardiopatía congénita infrecuente, que se reconoce fácilmente por la asociación de 2 o más lesiones cardíacas obstructivas en el corazón izquierdo. En adultos la mayoría de los casos descritos son formas incompletas (96\% según las series) ${ }^{(6)}$. Los pacientes suelen presentar una baja mortalidad en la vida adulta, pero con una substancial morbilidad relacionada con arritmias, insuficiencia cardíaca e intervenciones cardiovasculares.

El reconocimiento precoz de esta entidad mediante métodos por imágenes permite un seguimiento adecuado del paciente y una intervención precoz.

\section{Ideas para recordar}

- El complejo de Shone es una cardiopatía congénita infrecuente.

- Se caracteriza por presentar dos o más lesiones obstructivas en el corazón izquierdo, tanto a nivel del tracto de entrada como del tracto de salida.

- Las lesiones originalmente descritas fueron el anillo supravalvular mitral, la válvula mitral en "paracaídas", la estenosis subaórtica y la coartación aórtica.

- La mayoría de los pacientes requieren al menos una intervención quirúrgica a lo largo de su vida, siendo el tratamiento de la coartación aórtica y de la válvula mitral los más frecuentes.

- El grado de enfermedad mitral y la presencia de hipertensión pulmonar son los principales marcadores pronósticos post quirúrgicos.

\section{Bibliografía}

1. Shone JD, Sellers RD, Anderson RC, et al. The developmental complex of "parachute mitral valve," supravalvular ring of left atrium, subaortic stenosis, and coarctation of aorta. Am J Cardiol. 1963;11:714-725. doi:10.1016/00029149(63)90098-5.

2. True A, Baidya M, Lui C, Kilimnik G, Sadler M. Computed tomography imaging characteristics of Shone syndrome. Radiol Case Rep. 2018;14(2):164-167. doi:10.1016/j.radcr.2018.10.004.

3. Brauner RA, Laks H, Drinkwater DC Jr, et al. Multiple left heart obstructions (Shone's anomaly) with mitral valve involvement: long-term surgical outcome. Ann Thorac Surg. 1997 Sep;64(3):721-9.

4. Brown JW, Ruzmetov M, Vijay P, et al. Operative results and outcomes in children with Shone's anomaly. Ann Thorac Surg. 2005 Apr;79(4):1358-65.

5. López-Pardo F, Urbano-Moral JA, González-Calle A, et al. Three-Dimensional Transesophageal Echocardiography in the Anatomical Assessment of Isolated Parachute Mitral Valve in an Adult Patient. Echocardiography. 2015;32(11):1732-1735. doi:10.1111/echo.13003.

6. Aslam S, Khairy P, Shohoudi A, et al. Shone Complex: An Under-recognized Congenital Heart Disease With Substantial Morbidity in Adulthood. Can J Cardiol. 2017;33(2):253-259. doi:10.1016/j.cjca.2016.09.005.

7. Hakim FA, Kendall CB, Alharthi M, Mancina JC, Tajik JA, Mookadam F. Parachute mitral valve in adults-a systematic overview. Echocardiography. 2010;27(5):581-586. doi:10.1111/j.1540-8175.2009.01143.x 